\title{
Functional Assessment of the Collateral-Dependent Circulation in Chronic Total Coronary Occlusion Using Transthoracic Doppler Ultrasound and Venous Adenosine Infusion
}

\author{
Francesco Pizzuto, MD ${ }^{\mathrm{a}, *}$, Paolo Voci, MD, PhD ${ }^{\mathrm{a}}$, Paolo Emilio Puddu, $\mathrm{MD}^{\mathrm{b}}$, \\ Gaetano Chiricolo, MD ${ }^{\mathrm{a}}$, Mauro Borzi, MD ${ }^{\mathrm{a}}$, and Francesco Romeo, MD
}

\begin{abstract}
The measurement of collateral flow reserve (CFR; the hyperemic/baseline collateral flow velocity ratio) in patients with chronic total coronary occlusion requires invasive and expensive techniques. Noninvasive transthoracic coronary Doppler echocardiography may be an alternative option. Fifty-one patients with chronic total coronary occlusion were evaluated by transthoracic coronary Doppler echocardiography and venous adenosine infusion to measure CFR in occluded coronary arteries (the left anterior descending artery in 44 patients and the artery supplying the posterior descending artery in 7 patients). CFR data were plotted against 3 angiographic parameters: (1) grade of the epicardial filling of the occluded artery $(1=$ absent, $2=$ partial, $3=$ complete $)$, (2) stenosis of the donor artery, and (3) the extent of coronary artery disease (vessels with $\geq 70 \%$ stenosis). Collateral flow was maintained at stress in 34 patients (CFR $\geq 1$, range 1.0 to 2.2) but was withdrawn in 17 patients (CFR $<1$, range 0.25 to 0.90 ). CFR increased with the degree of angiographic collateral flow (grade 1: $0.73 \pm 0.29$; grade 2: $1.16 \pm 0.31$; grade 3: $1.34 \pm 0.49 ; \mathrm{F}=5.31$, $\mathrm{p}=0.008$ ). A multivariate model of CFR prediction showed a direct relation with angiographic collateral grade and the number of diseased vessels and an inverse relation with stenosis of the donor artery. In conclusion, CFR measurement is feasible by transthoracic coronary Doppler echocardiography. One third of the patients with chronic total coronary occlusion had collateral flow withdrawal at stress, which occurs when collateral circulation is poor and when the donor artery is stenotic. CFR correlates with angiographic collateral grade and with the extent of coronary artery disease. (C) 2006 Elsevier Inc. All rights reserved. (Am J Cardiol 2006;98:197-203)
\end{abstract}

Recently, transthoracic coronary Doppler echocardiography (TDE) has been introduced and validated for the noninvasive prediction of coronary artery disease,${ }^{1-4}$ the assessment of coronary recanalization in acute myocardial infarction, 5 and the evaluation of therapeutic coronary interventions. ${ }^{6-8}$ TDE can also predict left anterior descending (LAD) coronary occlusion on the basis of reversed flow in the epicardial artery ${ }^{9}$ and in its perforating branches. ${ }^{10} \mathrm{We}$ undertook this study to (1) assess the feasibility of TDE to noninvasively measure coronary flow reserve of the collateral-dependent circulation distal to the occluding lesion and (2) determine the relation of collateral flow reserve (CFR) to the angiographic collateral anatomy and flow.

\section{Methods}

Study population: We enrolled 56 consecutive patients (46 men, 10 women, mean age $59 \pm 9$ years, range 43 to 75 ) with chronic total occlusion of the LAD ( 44 patients) and of

\footnotetext{
aDepartment of Cardiology, "Tor Vergata" University; and bepartment of Heart and Great Vessels "A. Reale," "La Sapienza" University, Rome, Italy. Manuscript received September 24, 2005; revised manuscript received and accepted January 19, 2006.

* Corresponding author: Tel: 0039-06-86210144; fax: 0039-06-20904043.

E-mail address: francesco.pizzuto@uniroma1.it (F. Pizzuto).
}

the artery supplying the posterior descending coronary artery (PD; 12 patients). Inclusion criteria were (1) Thrombolysis In Myocardial Infarction anterograde coronary flow grade 0 , (2) normal global and regional left ventricular function at rest (wall motion score index $=$ 1), and (3) the absence of Q-wave myocardial infarction. Patients with contraindications to adenosine administration were excluded. All patients were in sinus rhythm and fasting state and provided written informed consent. All coronary active medications were withdrawn the day before the Doppler study. Figure 1 shows the clinical model that we used: occluded coronary artery (either the LAD or the artery supplying the PD), visible collaterals toward the occluded artery, and good left ventricular function.

TDE: TDE was performed as previously described in a modified 2-chamber view, ${ }^{4,6}$ using Sequoia C256 and C512 ultrasound systems (Siemens-Acuson, Mountain View, California). The LAD was scanned by a dedicated $7-\mathrm{MHz}$ transducer (3.5 MHz in color Doppler ultrasound) along the anterior interventricular groove, ${ }^{6}$ and the PD was scanned using a standard $3.5-\mathrm{MHz}$ transducer $(2 \mathrm{MHz}$ in color Doppler ultrasound). ${ }^{4}$ The best long-axis view in color Doppler imaging was obtained to optimize the angle be- 

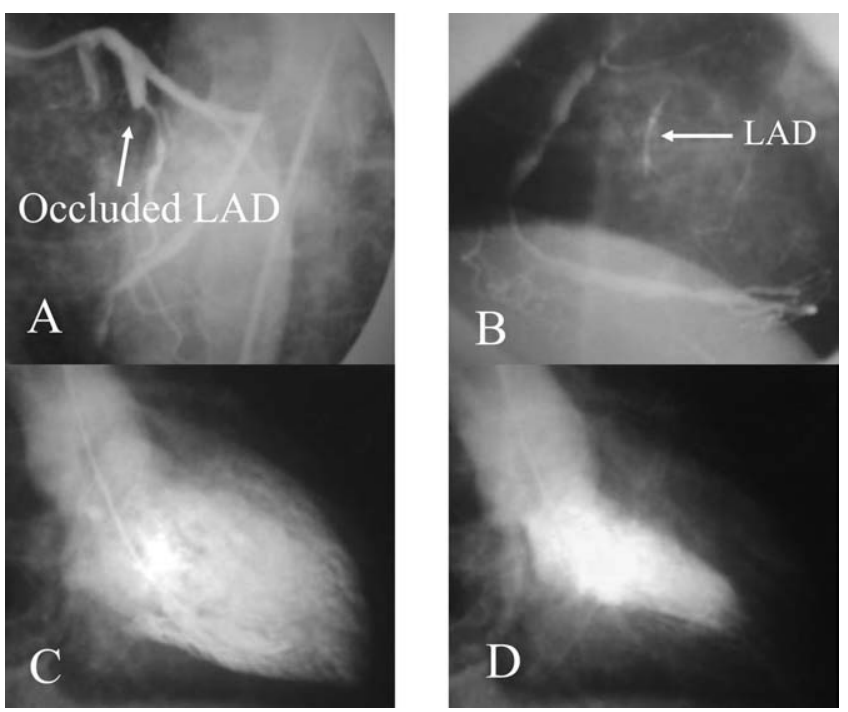

Figure 1. Clinical model of our study: occluded LAD coronary artery, without forward flow $(A)$, visible collaterals from the right coronary artery toward the occluded LAD $(B)$, and normal left ventricular function $(C, D)$.

tween flow and the Doppler beam. Imaging of the epicardial collaterals was attempted by angling the transducer lateral to the interventricular sulcus and imaging of the intramyocardial collaterals by focusing on perforating branches.

Coronary flow velocity and flow reserve: Flow direction in the occluded epicardial vessel was assessed by TDE and defined as normal (anterograde) or inverted (retrograde) (Figure 2). ${ }^{9}$ Similarly, flow direction in perforating branches was defined as normal (anterograde) or inverted (retrograde).${ }^{10}$ Coronary flow velocity was measured by pulsed Doppler ultrasound under color-coding guide. Coronary flow reserve of the collateral-dependent circulation was measured as the ratio between hyperemic and baseline diastolic velocities of the occluded artery. Blood flow velocities were recorded at baseline and during venous adenosine infusion (140 $\mu \mathrm{g} / \mathrm{kg} / \mathrm{min}$ over 90 seconds), within 24 to 48 hours of diagnostic coronary angiography. Peak and mean diastolic flow velocities were measured on the diastolic phase of the pulsed Doppler ultrasound curve. All studies were continuously recorded on S-VHS videotape for offline analysis. Heart rate was continuously monitored. Blood pressure was recorded at baseline, during adenosine infusion, and at recovery. For each test, the 3 greatest Doppler velocities were computed and averaged. CFR was calculated by the same operator performing the test, blind to the angiographic data, using peak diastolic values.

Angiography: Patients underwent left-sided cardiac catheterization with biplane left ventricular and coronary angiography. Coronary occlusion was estimated as the focal absence of anterograde flow. Angiographic collateral flow was determined according to the Rentrop classification, ${ }^{11}$ which takes into consideration the degree of the epicardial filling of the occluded artery $(1=$ filling of small side branches, 2 $=$ filling of major side branches with partial filling of the main epicardial vessel, $3=$ total filling of the main epicardial vessel). Patients were grouped according to angiographic collateral grade: group 1: grade 1 collaterals; group 2: grade 2 collaterals; and group 3: grade 3 collaterals. The sources of collaterals (single or multiple) and the direction of collateral flow in the occluded artery (anterograde, retrograde, or mixed) were also considered and computed. The described characteristics of the collateral circulation were analyzed independently by 2 observers unaware of the Doppler results, and a consensus was reached in cases of disagreement. The interaction of the donor artery with CFR was assessed according to its anatomy proximal to the takeoff of the collateral vessels, stratifying the patients as follows: no significant stenosis ( $0 \%$ to $49 \%)$, intermediate stenosis (50\% to 69\%), and significant stenosis ( $\geq 70 \%$ ). In case of multiple sources of collaterals, the artery with the greatest stenosis was considered. We also considered the

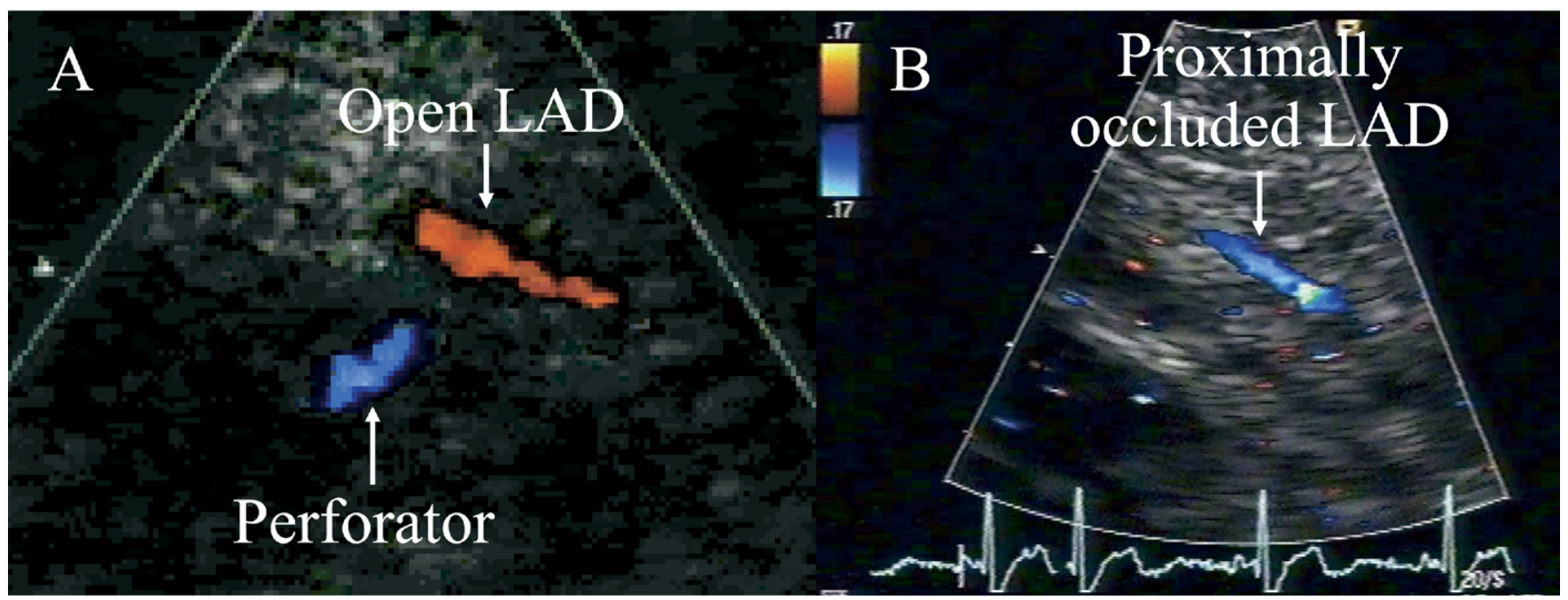

Figure 2. (A) Color flow Doppler ultrasound of a subject with normal anterograde flow either in the distal LAD coronary artery (red) or in a perforator (blue). (B) Patient with occluded LAD and inverted flow in the distal tract of the artery (blue) on color Doppler ultrasound. 
Table 1

Study population, risk factors, and hemodynamic and angiographic data

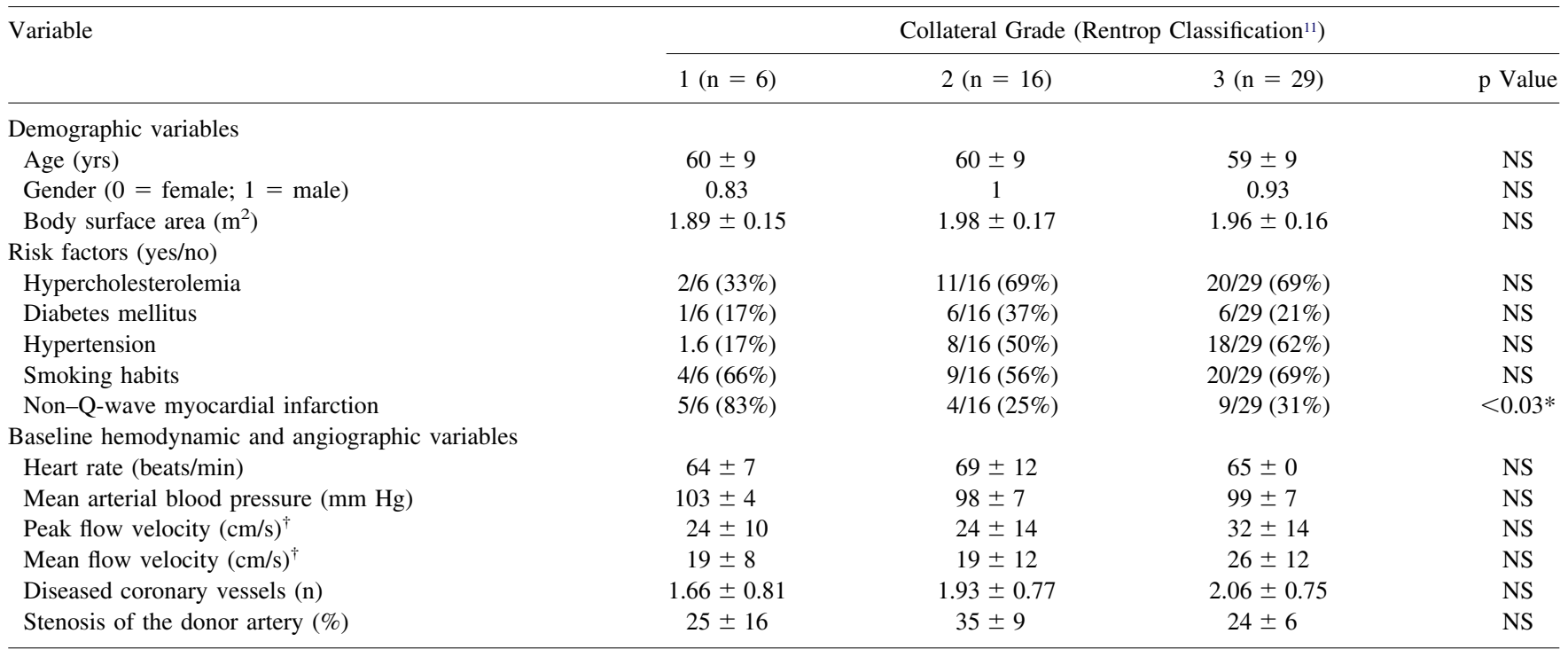

Risk factors $(0=$ absent, $1=$ present, based on standard cut-off values).

One-factor analysis of variance was used to test significance. In presence of a significant $(\mathrm{p}<0.05) \mathrm{F}$ test, pairwise comparisons were performed using Bonferroni's correction.

$* \mathrm{p}<0.05$ collateral grade 1 versus 2 .

${ }^{\dagger}$ Epicardial coronary artery.

impact on CFR of the extent of coronary artery disease, computing the number of remote arteries with $\geq 70 \%$ luminal narrowing.

Statistical analysis: Data are expressed as mean \pm SD, except for data expressed as percentages or proportions, for which SEs are used. Only average data are presented for dichotomous $(0=$ absent, $1=$ present $)$ variables. One-way analysis of variance using Bonferroni's correction was used to analyze data when assessing intergroup differences (BMDP-7D, University of California Press, Berkeley, California). Two-way analysis of variance was used to analyze hemodynamic changes (basal vs adenosine) as distributed among groups (BMDP-2V, University of California Press). A linear multivariate analysis (all possible subsets regression; BMDP-9R, University of California Press) was performed to evaluate the impact on CFR (as a continuous dependent variable, $y$ ) of the following independent covariates $\left(\mathrm{x}_{1}\right.$ to $\left.\mathrm{x}_{\mathrm{n}}\right)$ : gender, age, collateral circulation type (based on Rentrop classification, ${ }^{11}$ coded 1 to 3 ), percentage stenosis of the donor artery (from $0 \%$ to $99 \%$ ), number of diseased vessels (coded 1 to 3), coronary risk factors (hypercholesterolemia [blood cholesterol $\geq 240 \mathrm{mg} / \mathrm{dl}$ ], diabetes [blood glucose $\geq 128 \mathrm{mg} / \mathrm{dl}$ ], hypertension [systolic and/or diastolic blood pressure $\geq 140$ or $90 \mathrm{~mm} \mathrm{Hg}$ )], and smoking [ $>5$ cigarettes/day]; $0=$ absent, $1=$ present), and the presence of non-Q-wave myocardial infarction. Multiple $\mathrm{r}$ and $\mathrm{r}^{2}$ values were calculated, and coefficient and SE $(t)$ was considered to assess the significance of covariates included in the model. A value of $p<0.05$ was considered statistically significant.

\section{Results}

Feasibility and patients characteristics: Adequate baseline and hyperemic Doppler recordings of the collateraldependent circulation were obtained in 51 of 56 patients (92\%): 44 of 44 in the LAD and 7 of 12 in the PD. There were no statistically significant differences among groups regarding gender, body surface area, coronary risk factors, and baseline hemodynamic factors, whereas there was a greater prevalence of non-Q-wave myocardial infarction in remote areas in patients with grade 1 collaterals (Table 1).

Angiographic characteristics: According to angiographic collateral grade, 6 patients (12\%) had grade 1, $16(31 \%)$ grade 2, and 29 (57\%) grade 3 collateral flow. Thirty-seven patients $(72 \%)$ had single sources of collaterals, whereas 14 (28\%) had multiple sources. Fifteen patients (30\%) had single-vessel disease (i.e., the occluded coronary artery), 14 (27\%) had 2-vessel disease, and $22(43 \%)$ had 3-vessel disease (mean vessel disease $1.98 \pm 0.75$ ). Regarding the anatomy of the donor artery proximal to the takeoff of the collateral vessels, 32 patients had no significant stenosis (average percentage stenosis $3.12 \pm 1.76 \%$ ), 8 had intermediate stenosis $(53.75 \pm 1.83 \%)$, and 11 had significant stenosis $(79.1 \pm 2.5 \%)$. Collateral flow was anterograde in 21 patients, retrograde in 16, and mixed (anterograde and retrograde) in 14 .

Collateral flow and flow reserve: On TDE, collateral flow in the epicardial occluded artery was anterograde in 28 patients (55\%), retrograde in 22 (43\%; Figure 3), and mixed in 1 , whereas intramyocardial flow, as detected in perforat- 


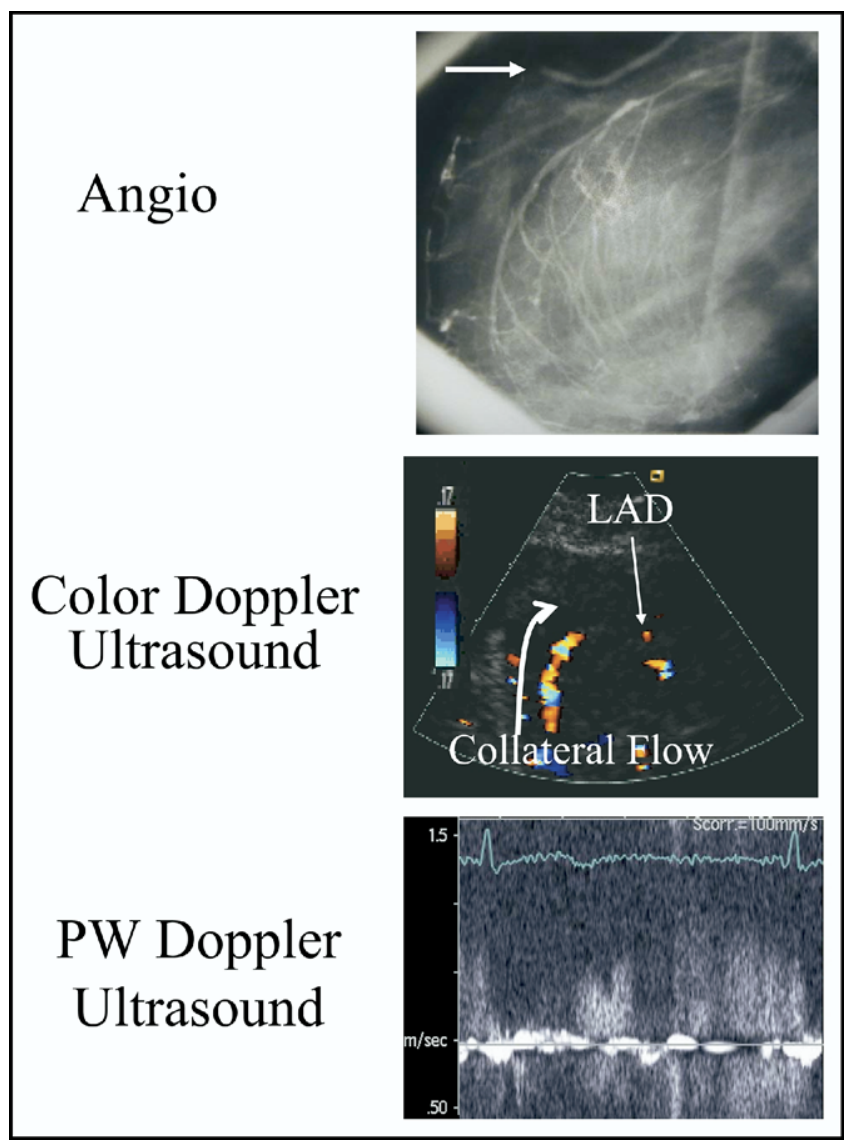

Figure 3. Top panel, coronary angiography of a patient with proximally occluded LAD coronary artery and anterograde flow, supplied by huge collaterals from the right coronary artery. Arrow, the tip of the coronary catheter, placed in the right coronary ostium. Middle panel, a large collateral is visible by color Doppler ultrasound. Flow in the LAD is anterograde (red). Bottom panel, pulsed Doppler wave ultrasound shows high-velocity $(1 \mathrm{~m} / \mathrm{s})$ continuous collateral flow.

ing branches, was inverted in 20 patients (40\%), normal in $22(43 \%)$, and undetectable in $9(17 \%)$. A direct epicardial collateral connection was visualized in 12 patients (24\%), by color TDE (Figure 3), and the corresponding spectral Doppler ultrasound always showed a chaotic, high-velocity $(>50 \mathrm{~cm} / \mathrm{s})$ flow signal (Figure 3). Collateral-dependent flow reserve was $<2$ in most of the patients (45 of 51). Collateral flow withdrawal at stress (CFR $<1$; Figure 4) was observed in 17 patients (30\%). CFR significantly increased with the grade of angiographic collateral flow (Figure 5): it was $0.73 \pm 0.29$ in grade $1,1.16 \pm 0.31$ in grade 2 , and $1.34 \pm$ 0.49 in grade $3(\mathrm{~F}=5.31, \mathrm{p}=0.008$; Figures 6 and 7$)$. Multiple sources of collaterals slightly but nonsignificantly increased CFR (single source $=1.15 \pm 0.47$, multiple sources $=1.37 \pm 0.39 ; \mathrm{F}=2.21, \mathrm{p}=0.14$; Figure 8).

Multivariate prediction of CFR: In the 51 patients with adequate Doppler recordings, CFR was $1.21 \pm 0.46$ (range 0.25 to 2.27; coefficient of variation 0.38 ). The prediction of CFR was good (multiple $\mathrm{r}=0.65$, multiple $\mathrm{r}^{2}=0.42, \mathrm{~F}=$ $8.28, \mathrm{p}<0.00001)$ on the basis of independent variables considered. However, only percentage stenosis of the donor

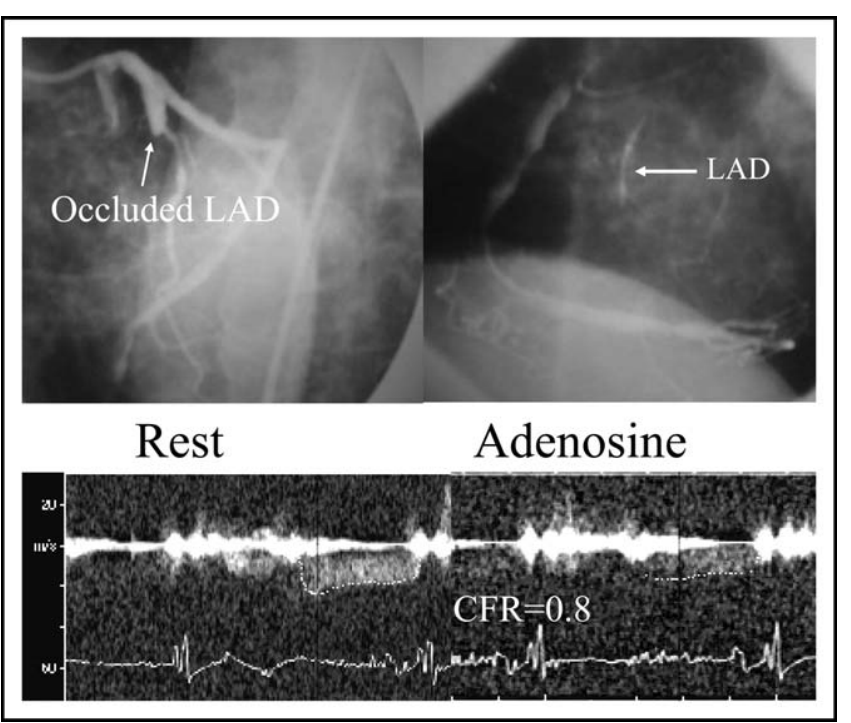

Figure 4. Top panel, coronary angiography of a patient with proximally occluded LAD coronary artery and Rentrop grade 1 collaterals from the right coronary artery. Bottom panel, flow in the LAD measured by transthoracic Doppler ultrasound is retrograde, and CFR is 0.8 .

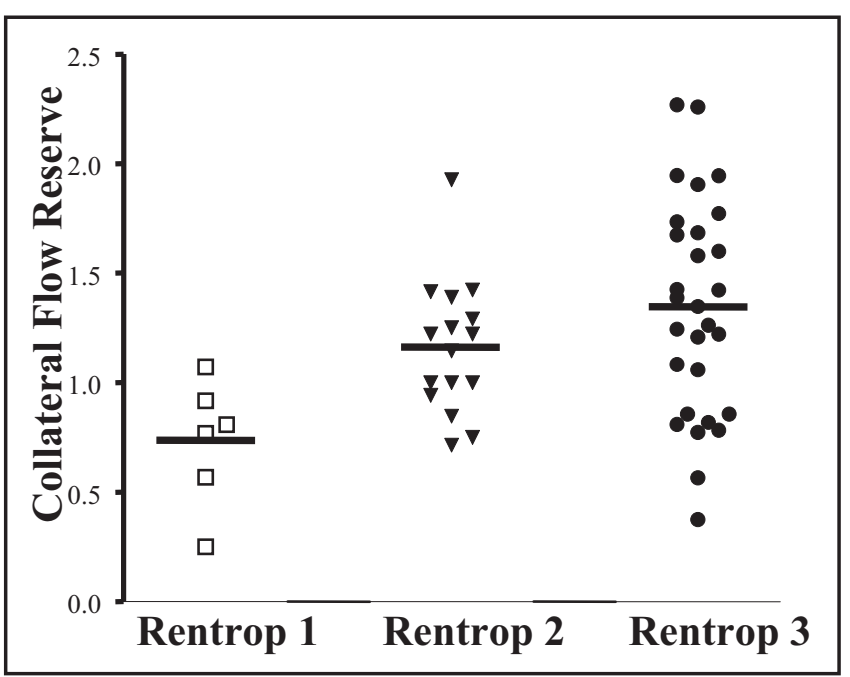

Figure 5. CFR of the 51 patients with chronic total occlusion of the coronary artery grouped according to Rentrop collateral flow classification.

artery (coefficient $-0.0047, t=-2.63, \mathrm{p}<0.012$ ), the number of diseased vessels (coefficient $0.27, t=3.27$, $\mathrm{p}<0.002$ ), and the type of collateral circulation (coefficient $0.21, t=2.75, \mathrm{p}<0.009)$ did contribute significantly. The multivariate intercept was 0.23 . Therefore, to predict CFR in a patient with $30 \%$ stenosis of the donor artery (proximal to the takeoff of the collateral branch), 3-vessel coronary artery disease, and Rentrop 2 collateral circulation, the back application of these multivariate results gives an approximate CFR $=$ $0.23+[(-0.0047 \times 30+(0.27 \times 3)+(0.21 \times 2)]=1.32$. In contrast, in a patient with $70 \%$ stenosis of the donor artery, 2-vessel coronary artery disease, and Rentrop 1 collateral circulation, $\mathrm{CFR}=0.23+[(-0.0047 \times 70)+(0.27 \times 2)+$ $(0.21 \times 1)]=0.65$. Thus, in this model, the greater the stenosis of the donor artery, the smaller the CFR, whereas 


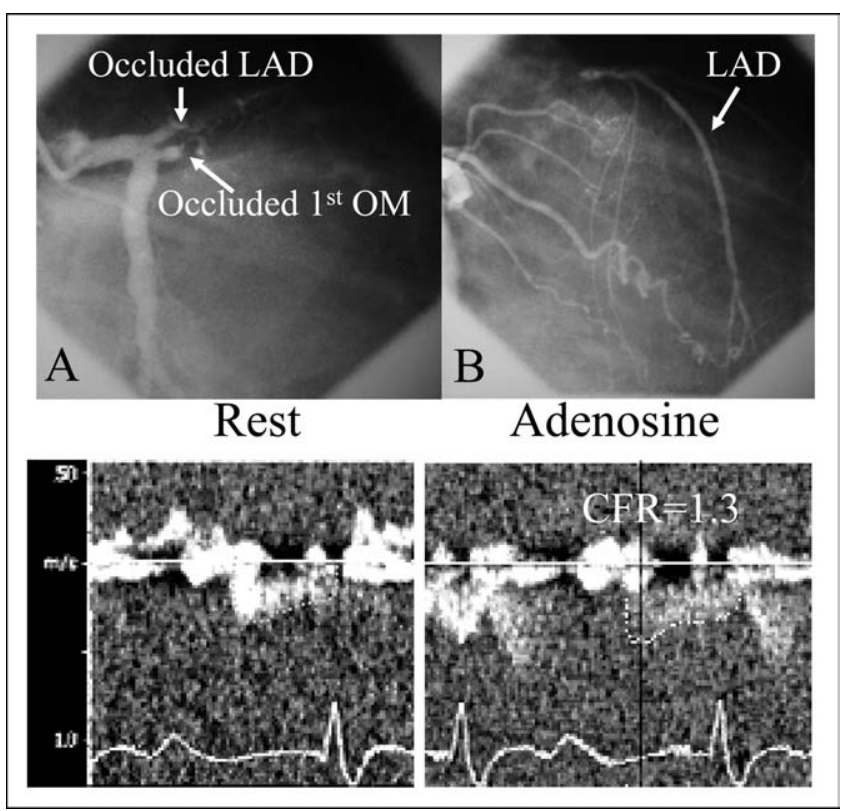

Figure 6. Top panel, coronary angiography of a patient with proximally occluded LAD and first obtuse marginal (OM 1) coronary arteries (A). Retrograde collateral flow is provided by a direct connection between the right coronary artery and the LAD $(B)$. Bottom panel, flow in the LAD measured by transthoracic Doppler ultrasound is retrograde, and CFR is 1.3 .

the larger the number of diseased vessels and the greater the collateral grade, the greater the CFR.

Hemodynamic variables: Adenosine infusion induced similar changes in all groups of heart rate, which increased $(\mathrm{p}<0.0001)$, and of mean arterial pressure, which decreased ( $\mathrm{p}<0.0001)$. Two-way analysis of variance interaction terms were $p=0.13$ and $p=0.59$ for heart rate and mean arterial pressure changes, respectively. Moreover, there was no statistical difference between rate-pressure product at rest $(6,608 \pm 795$ for collateral grade $1,6,816 \pm 1,409$ for collateral grade 2, and 6,428 \pm 969 for collateral grade 3) and during adenosine infusion $(6,820 \pm 1,270$ for collateral grade 1, 6,344 $\pm 1,195$ for collateral grade 2, and 6,460 \pm 1,189 , for collateral grade 3 ).

Variability: To check intraobserver variability, 1 operator duplicated measurements of baseline and adenosine peak diastolic velocities in frozen, digital images from 51 patients. To check interobserver variability, a second observer made the same measurements, unaware of the results from the previous analysis. Variability was computed by calculating the technical error (coefficient of variability) for duplicate velocity measurements and for coronary flow reserve. We used the formula sigma $=\sqrt{\left[\operatorname{sum}\left(x_{1}-x_{2}\right) * * 2 /\right.}$ $(2 \mathrm{n}-1)]$, and variability was expressed as a fraction of the mean from all duplicate observations. Intraobserver variability $(2 \mathrm{n}-1=101)$ for baseline velocity, adenosine velocity, and coronary flow reserve was $3.49 \%( \pm 1.02$ $\mathrm{cm} / \mathrm{s}), 2.90 \%( \pm 1.10 \mathrm{~cm} / \mathrm{s})$, and $2.15 \%( \pm 0.026 \mathrm{U})$, respectively. Interobserver variability $(2 \mathrm{n}-1=101)$ for baseline velocity, adenosine velocity, and coronary flow reserve was $3.57 \%( \pm 1.05 \mathrm{~cm} / \mathrm{s}), 3.38 \%( \pm 1.29 \mathrm{~cm} / \mathrm{s})$ and $2.74 \%$ $( \pm 0.033 \mathrm{U})$, respectively.

\section{Discussion}

This study shows for the first time that the function of collateral-dependent circulation is measurable with TDE. Our findings are similar to those recently reported by Werner at $\mathrm{al}^{12}$ by intracoronary Doppler ultrasound, with the difference that they found collateral flow withdrawal mainly in patients with Rentrop grade 2 collaterals rather than grade 1 (Figure 5). We may therefore suggest that a poorly developed collateral circulation is more prone than a well-developed collateral circulation to withdraw its flow support to the occluded coronary artery under stress. Unfortunately, our population contained few patients in grade 1 collaterals to confirm this finding.

Werner et al ${ }^{12}$ also found small baseline Doppler velocities (approximately $10 \mathrm{~cm} / \mathrm{s}$ ) in the collateral-dependent circulation in most patients, whereas we consistently

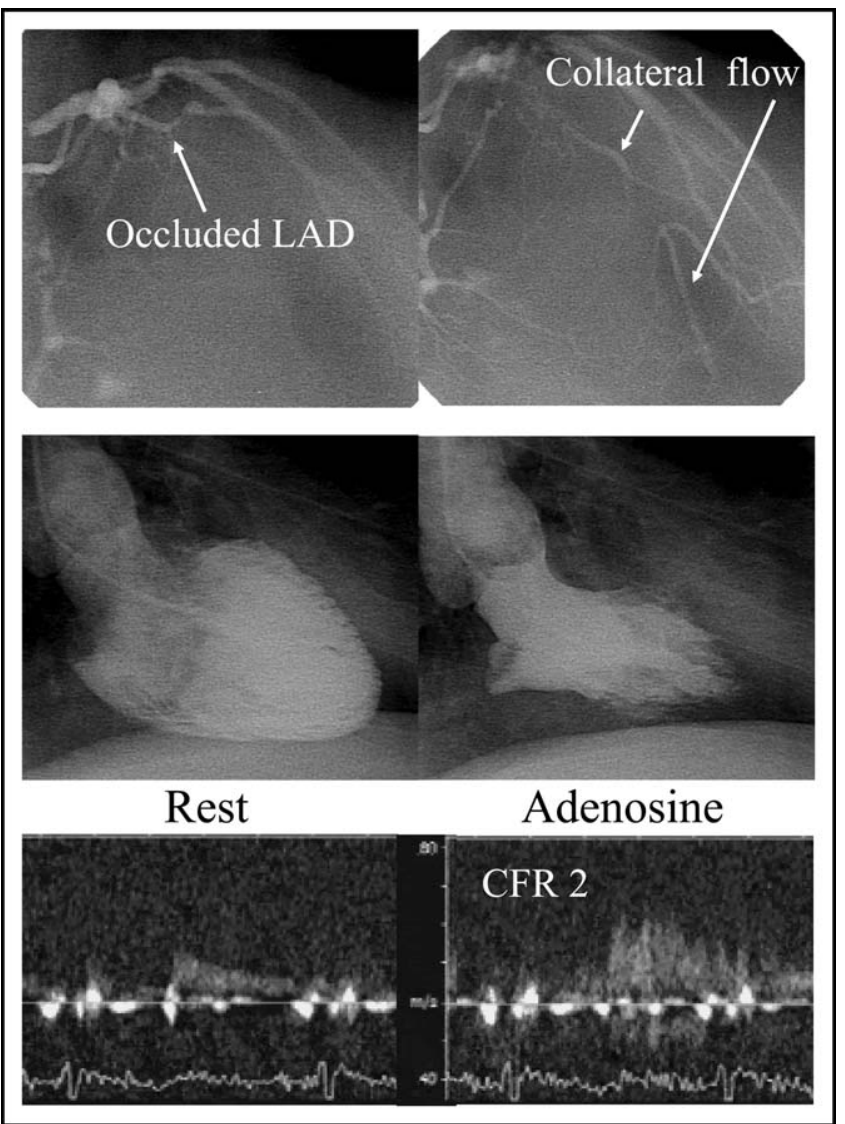

Figure 7. Top panel, coronary angiography of a patient with proximally occluded LAD coronary artery. Anterograde collateral flow is provided by multiple connections between the left circumflex coronary artery and the LAD (Rentrop grade 3). The right coronary artery is also occluded and receives collaterals from the circumflex. Middle panel, left ventriculography shows normal left ventricular function. Bottom panel, pulsed Doppler ultrasound shows anterograde LAD flow, and CFR is 2 . 


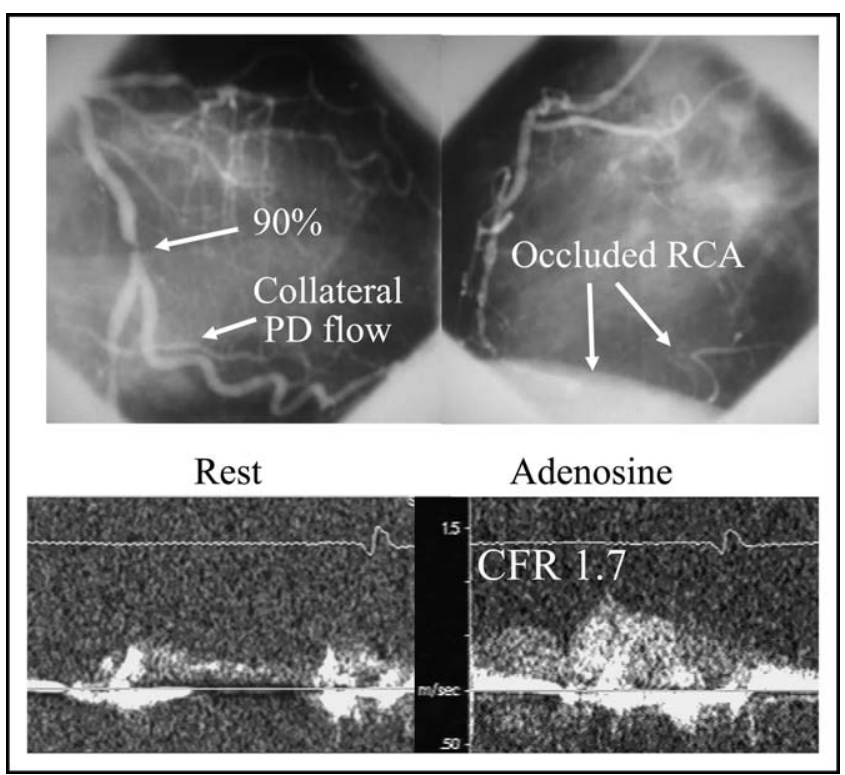

Figure 8. Top panel, coronary angiography of a patient with occlusion of the distal tract of the right coronary artery (RCA). The collateral flow feeding the PD and posterolateral coronary arteries (Rentrop grade 3) is supplied by the proximal portion of the RCA and by septal branches of the LAD coronary artery (a combination of anterograde and retrograde collateral flows, supplied by multiple sources of collaterals). The patient had also a tight stenosis of the circumflex coronary artery (arrow). Bottom panel, the CFR, assessed by transthoracic Doppler ultrasound in the PD coronary artery, is 1.7 .

found normal velocities, similar to those reported by Watanabe et $\mathrm{al}^{9}(26.4 \pm 9.8$ and $28.1 \pm 7.4 \mathrm{~cm} / \mathrm{s}$, respectively).

Therefore, it appears to be a difference between invasive and noninvasive studies. A possible explanation may be that Werner et al $^{12}$ included patients with previous myocardial infarctions of the index artery, whereas Watanabe et $\mathrm{al}^{9}$ and we studied only patients without previous infarctions of the myocardium distal to the occluded artery. In addition, there may be some pitfalls in the measurement of collateral flow by intracoronary Doppler flow wire: (1) it is technically demanding, (2) the position of the tip of the wire relative to the occlusion and to the input of collateral flow may be suboptimal, (3) sampling too close to the occlusion site may result in small coronary flow velocity recordings, (4) artifacts may be generated by guidewire motion and inadvertent contact with the vessel wall, ${ }^{13}$ and (5) placing the Doppler wire requires a previous opening of the vessel with a stiff wire, which creates a new channel, potentially altering collateral flow dynamics. Conversely, TDE measurements are obtained in a more physiologic, no-touch way, in the distal tract of the vessel, in which coronary flow velocity likely recovers to its normal value.

Several investigators have interpreted the reduction of collateral flow at stress in patients with chronic total coronary occlusion as "coronary steal." 12,14 However, because collateral flow toward the occluded artery is actually a gift of the "donor" artery, the accusation of theft is unfair, and the term "coronary flow withdrawal" may be more appropriate.

Recent studies with TDE have shown that LAD occlusion was invariably associated with retrograde flow $^{9}$ in the index artery and/or in its perforating branches. ${ }^{10}$ However, angiographic experience teaches that collateral flow may be anterograde, retrograde, or mixed. In fact, in our patients with chronic total coronary occlusion, inverted flow in the distal LAD was found at TDE in only $43 \%$ of the patients, whereas preserved anterograde flow was found in $55 \%$. Flow direction was undetectable in the remaining $2 \%$. Similarly, normal intramyocardial anterograde flow direction was preserved in more than half of the cases. Nevertheless, the TDE finding of retrograde diastolic flow, either in the epicardial vessel or in perforating branches, is a useful and specific sign of coronary occlusion. The reasons for the discrepancy between our data and those of others ${ }^{9,10}$ are not clear, but patient selection, racial difference in chest wall acoustic impedance, and coronary flow sampling site by TDE may play a role. In our population, there was also some discrepancy between the direction of flow when detected at angiography or by TDE. This is mainly due to the high prevalence of mixed flow $(n=14)$ at angiography that may be classified by TDE as either retrograde or anterograde, because ultrasound still has no panoramic view of the coronary tree.

An additional peculiar finding of the collateral circulation detected by TDE is the high blood flow velocity in the collateral pathways (Figure 3). In fact, collateral vessels have an intrinsic resistance to flow that is comparable to a $70 \%$ coronary artery stenosis. ${ }^{15}$ This resistance may produce flow acceleration at rest (as a mechanism to keep the coronary output constant) and may prevent the adequate increase of flow at stress in the recipient artery $(\mathrm{CFR}<2)$, as found in most of our cases with chronic total coronary occlusion. Interestingly, coronary flow reserve $<2$ also characterizes patients with significant coronary artery stenosis. ${ }^{16,17}$ Unfortunately, direct imaging of the collateral pathways, using the available TDE technology, is feasible in only $1 / 4$ of the cases.

A direct relation between the resistance of the donor artery and the ability of the collateral-dependent circulation to increase flow during vasodilation has been found using intracoronary Doppler ultrasound and pressure wires. ${ }^{12,14}$ In our study, we did not measure the resistances but evaluated the degree of the stenosis of the donor coronary artery proximal to the collateral takeoff, which inversely correlated with the CFR (the greater the stenosis, the smaller the CFR). An interesting new finding of our study is that CFR proportionally increased with the number of diseased vessels, suggesting a chronic stimulus to create more connections within the myocardium in case of diffuse atherosclerotic narrowing of the epicardial coronary arteries.

1. Hozumi T, Yoshida K, Akasaka T, Asami Y, Ogata Y, Takagi T, Kaji S, Kawamoto T, Ueda Y, Morioka S. Noninvasive assessment of 
coronary flow velocity and coronary flow velocity reserve in the left anterior descending coronary artery by Doppler echocardiography. J Am Coll Cardiol 1998;32:1251-1259.

2. Caiati C, Montaldo C, Zedda N, Montisci R, Ruscazio M, Lai G, Cadeddu M, Meloni L, Iliceto S. Validation of a new noninvasive method (contrast-enhanced transthoracic second harmonic echo Doppler) for the evaluation of coronary flow reserve: comparison with intracoronary Doppler flow wire. J Am Coll Cardiol 1999;34:11931200.

3. Caiati C, Montaldo C, Zedda N, Bina A, Iliceto S. New noninvasive method for coronary flow reserve assessment. Contrast-enhanced transthoracic second harmonic echo Doppler. Circulation 1999;99: 771-778.

4. Voci P, Pizzuto F, Mariano E, Puddu PE, Chiavari PA, Romeo F. Measurement of coronary flow reserve in the anterior and posterior descending coronary arteries by transthoracic Doppler ultrasound. Am J Cardiol 2002;90:988-991.

5. Voci P, Mariano E, Pizzuto F, Puddu PE, Romeo F. Coronary recanalization in anterior myocardial infarction. The open perforator hypothesis. J Am Coll Cardiol 2002;40:1205-1213.

6. Pizzuto F, Voci P, Mariano E, Puddu PE, Sardella G, Nigri A. Assessment of flow velocity reserve by transthoracic Doppler echocardiography and venous adenosine infusion before and after left anterior descending coronary artery stenting. J Am Coll Cardiol 2001;38:155162.

7. Pizzuto F, Voci P, Mariano E, Puddu PE, Chiavari PA, Romeo F. Noninvasive coronary flow reserve assessed by transthoracic coronary Doppler ultrasound in patients with left anterior descending coronary artery stents. Am J Cardiol 2003;91:522-526.

8. Pizzuto F, Voci P, Mariano E, Puddu PE, Aprile A, Romeo F. Evaluation of flow in the left anterior descending coronary artery but not in the left internal mammary artery graft predicts significant stenosis of the arterial conduit. J Am Coll Cardiol 2005;45:424-432.
9. Watanabe N, Akasaka T, Yamaura Y, Akiyama M, Koyama Y, Kamiyama N, Neishi Y, Kaji S, Saito Y, Yoshida K. Noinvasive detection of total occlusion of the left anterior descending coronary artery with transthoracic Doppler echocardiography. J Am Coll Cardiol 2001;38: $1328-1332$

10. Hirata K, Watanabe H, Hozumi T, Tokai K, Otsuka R, Fujimoto K, Shimada K, Muro T, Yoshiyama M, Yoshikawa J. Simple detection of occluded coronary artery using retrograde flow in septal branch and left anterior descending coronary artery by transthoracic Doppler echocardiography at rest. J Am Soc Echocardiogr 2004;17:108-113.

11. Rentrop KP, Cohen M, Blanke H, Phillips RA. Changes in collateral channel filling immediately after controlled coronary artery occlusion by an angioplasty balloon in human subjects. $J$ Am Coll Cardiol 1985;5:587-592.

12. Werner GS, Figulla HR. Direct assessment of coronary steal and associated changes of collateral hemodynamics in chronic total coronary occlusions. Circulation 2002;106:435-440.

13. van Liebergen RA, Piek JJ, Koch KT, de Winter RJ, Schotborgh CE, Lie KI. Quantification of collateral flow in humans: a comparison of angiographic, electrocardiographic and hemodynamic variables. $J$ Am Coll Cardiol 1999;33:670-677.

14. Seiler C, Fleisch M, Meier B. Direct intracoronary evidence of collateral steal in humans. Circulation 1997;96:4261-4267.

15. Flameng W, Schwarz F, Hehrlein FW. Intraoperative evaluation of the functional significance of coronary collateral vessels in patients with coronary artery disease. Am J Cardiol 1978;42:187-192.

16. Kern MJ, DeBruyne B, Pijls NHJ. From research to clinical practice: current role of physiologically based decision making in the catheterization laboratory. J Am Coll Cardiol 1997;30:613-620.

17. Voci P, Pizzuto F, Mariano E, Puddu PE, Sardella G, Romeo F. Usefulness of coronary flow reserve measured by transthoracic coronary Doppler ultrasound to detect severe left anterior descending coronary artery stenosis. Am J Cardiol 2003;92:1320-1324. 